\title{
A New Phase of GaN
}

\section{Qingyang Fan, ${ }^{1}$ Changchun Chai, ${ }^{1}$ Qun Wei, ${ }^{2}$ Jionghao Yang, ${ }^{3}$ Peikun Zhou, Dongyun Zhang, ${ }^{5}$ and Yintang Yang ${ }^{1}$}

\author{
${ }^{1}$ Key Laboratory of Ministry of Education for Wide Band-Gap Semiconductor Materials and Devices, School of Microelectronics, \\ Xidian University, Xian 710071, China \\ ${ }^{2}$ School of Physics and Optoelectronic Engineering, Xidian University, Xian 710071, China \\ ${ }^{3}$ Xi'an Institute of Applied Optics, Xi'an 710065, China \\ ${ }^{4}$ Faculty of Science, University of Paris-Sud, 91400 Paris, France \\ ${ }^{5}$ National Supercomputing Center in Shenzhen, Shenzhen 518055, China
}

Correspondence should be addressed to Qingyang Fan; qyfan_xidian@163.com

Received 24 December 2015; Revised 4 March 2016; Accepted 13 March 2016

Academic Editor: Zhongfang Chen

Copyright (C) 2016 Qingyang Fan et al. This is an open access article distributed under the Creative Commons Attribution License, which permits unrestricted use, distribution, and reproduction in any medium, provided the original work is properly cited.

\begin{abstract}
The structural, mechanical, and electronic properties of the orthorhombic GaN (Pnma-GaN) are investigated at ambient pressure by using first-principles calculations method with the ultrasoft pseudopotential scheme. The elastic constants and phonon calculations reveal Pnma-GaN is mechanically and dynamically stable at ambient pressure. The calculated Young modulus of Pnma-GaN is $170 \mathrm{GPa}$, which is the three-fifths of wurtzite-GaN. Electronic structure study shows that Pnma-GaN is a direct semiconductor with band gap of $1.847 \mathrm{eV}$. The anisotropic calculation shows that wurtzite-GaN has a smaller elastic anisotropy than that of Pnma$\mathrm{GaN}$ in Young's modulus. In addition, when the composition of aluminum increases from 0 to 0.063 in the alloy, the band gap decreases initially and increases afterward for Pnma- $\mathrm{Ga}_{1-x} \mathrm{Al}_{x} \mathrm{~N}$, while, for wurtzite- $\mathrm{Ga}_{1-x} \mathrm{Al}_{x} \mathrm{~N}$, the band gap increases with the increasing composition $x$. Due to the structural porous feature, Pnma-GaN can also be expected to be a good hydrogen storage material.
\end{abstract}

\section{Introductions}

A complete acquisition of the physical properties of materials from the macro to the atomic scale is compulsory for device applications. Consequently, theoretical and experimental efforts are beneficial to the study of major properties of main materials and to the search for new materials. Theoretical studies are built on systematic models and computer simulations. One of the most precise microscopic theories in computational materials science is density functional theory (DFT) [1], which sufficiently defines the ground state physical properties of electronic system. It is well known that the GaN single crystal can be considered as the best candidate for wide-gap UV detectors and photodiodes $[2,3]$. One of the best ways to improve their technology parameters in our opinion consists of the synthesis of an appropriate solid alloy to continuously vary their band energy gaps creating a continuous set of solid alloy. There have been many published articles of electronic and optical properties in $\mathrm{GaN}$ single crystal. The first band energy structure for wurtzite $\mathrm{Ga}_{1-x} \mathrm{Al}_{x} \mathrm{~N}(x=0.25,0.50$, and 0.75$)$ has been calculated by Rubio et al. [4]. Then, Malachowski et al. [5] have calculated the band energy structure of $\mathrm{Ga}_{1-x} \mathrm{Al}_{x} \mathrm{~N}(x=0.15$ and 0.35$)$ solid alloys. The band structure of the $\mathrm{Ga}_{1-x} \mathrm{Al}_{x} \mathrm{~N}(x=$ $0.15,0.52$, and 0.65 ) solid crystalline alloys was calculated by Kityk [6] first using another approach of calculations. This approach includes the use of the orthogonalisation procedure for the plane-wave basis set with respect to the core-like states and the applications of the PA screening procedure; and they found that this approach can be extended to different binary wide-gap semiconductors solid alloys during the estimation of the band energy structure. In [7], a good agreement was predicted with experimental results for the minimum of band gaps in the wurtzite-like $\mathrm{Ga}_{1-x} \mathrm{Al}_{x} \mathrm{~N}$ structure. For the zinc-blend structure, it was evaluated that GaN will have a direct band gap of $3.1 \mathrm{eV}$ at $\Gamma$ point in good agreement with recent reported experiments on cubic GaN crystals $(3.35 \mathrm{eV})$ [8]. Miwa and Fukumoto [9] calculated the 
TABLE 1: Calculated lattice constants for $\mathrm{Ga}_{1-x} \mathrm{Al}_{x} \mathrm{~N}(2 \times 1 \times 2$ supercell $)$ alloy.

\begin{tabular}{|c|c|c|c|c|c|c|c|}
\hline \multirow{3}{*}{ Space group } & \multirow{3}{*}{$\begin{array}{c}\text { Composition } \\
x\end{array}$} & \multicolumn{6}{|c|}{ Lattice constant $(\AA)$} \\
\hline & & \multicolumn{3}{|c|}{ GGA } & \multicolumn{3}{|c|}{ LDA } \\
\hline & & $a$ & $b$ & $c$ & $a$ & $b$ & $c$ \\
\hline \multirow{5}{*}{ Pnma } & 0 & 12.136 & 3.306 & 10.850 & 11.795 & 3.232 & 10.747 \\
\hline & 0.031 & 12.113 & 3.298 & 10.819 & 11.761 & 3.226 & 10.656 \\
\hline & 0.063 & 12.079 & 3.291 & 10.793 & 11.717 & 3.219 & 10.635 \\
\hline & 0.094 & 12.037 & 3.285 & 10.764 & 11.680 & 3.213 & 10.607 \\
\hline & 0.125 & 12.018 & 3.267 & 10.721 & 11.657 & 3.207 & 10.581 \\
\hline \multirow{5}{*}{$\mathrm{P}_{3} \mathrm{mc}$} & $0^{\mathrm{a}}$ & 3.225 & & 5.257 & 3.156 & & 5.148 \\
\hline & 0 & $3.232^{\mathrm{b}}$ & & 5.268 & $3.196^{\mathrm{c}}$ & & 5.222 \\
\hline & & & & & $3.145^{\mathrm{d}}$ & & 5.111 \\
\hline & 0 & & & & $3.190^{\mathrm{e}}$ & & 5.190 \\
\hline & Exp. ${ }^{f}$ & 3.190 & & 5.189 & & & \\
\hline
\end{tabular}

a This work, ${ }^{\mathrm{b}}[23],{ }^{\mathrm{c}}[24],{ }^{\mathrm{d}}[25],{ }^{\mathrm{e}}[26]$, and ${ }^{\mathrm{f}}[22]$.

GaN with wurtzite-like structure and zinc-blend structure using the first-principle pseudopotential calculations. Similar results were concluded in $[10,11]$.

Recently, we have found that Pnma-GaN is mechanically and dynamically stable at ambient pressure via elastic constants and phonon calculations. Previous work has not systematically investigated the structural and mechanical properties of the new Pnma phase of GaN and their $\mathrm{Ga}_{1-x} \mathrm{Al}_{x} \mathrm{~N}$ alloys. In this work, we have first detailed the equilibrium crystal structure, mechanical properties, and electronic properties of GaN based on DFT.

\section{Computational Methods}

The self-consistent calculations were performed using density functional theory (DFT) $[1,12]$ within the Perdew-BurkeErnzerhof (PBE) exchange-correlation and employing the generalized gradient approximation (GGA) [13] and the local density approximation (LDA) $[14,15]$ as implemented in the Cambridge Serial Total Energy Package (CASTEP) code [16]. A plane-wave basis set with energy cut-off of $380 \mathrm{eV}$ was used for Pnma-GaN and their $\mathrm{Ga}_{1-x} \mathrm{Al}_{x} \mathrm{~N}$ alloys in these calculations. For wurtzite-GaN, we used a plane-wave basis set with energy cut-off $400 \mathrm{eV}$, which is used with GGA and LDA. The Monkhorst-Pack [17] $k$-point separation with a grid of $0.025 \AA^{-1}$ along the three axes in the reciprocal space was used in all calculations. The Broyden-FletcherGoldfarb-Shanno (BFGS) [18] minimization scheme was used in geometry optimization. The total energy convergence tests showed that convergence to less than $1 \mathrm{meV} /$ atom was achieved with the above calculation parameters. All energy on atoms converged to less than $1 \mathrm{meV} /$ atom for the geometry relaxation; and all the components of the Hellmann-Feynman stress tensor were reduced to the order of $0.01 \mathrm{GPa}$ at the given pressure. The elastic constants were determined by applying a set of given homogeneous strains with a finite value $(0.1 \%)$ to the equilibrium lattice and calculating the resulting stress according to optimizing the structure under the strain. The bulk modulus, shear modulus, and Young's modulus were estimated by employing Voigt-Reuss-Hill approximation [19-21]. The phonons spectra of Pnma-GaN are using the linear response approach, or the so-called density functional perturbation theory (DFPT) is one of the most popular methods of ab initio calculation of lattice dynamics [22].

\section{Results and Discussion}

The calculated lattice parameters of Pnma-GaN are presented in Table 1. Similarly, it is recognized within DFT that the lattice parameters of Pnma-GaN and wurtzite-GaN calculations are commonly underestimated for both GGA and LDA, while the LDA values are close to the experimental values for wurtzite-GaN, so LDA method can obtain more accurate results. The calculated lattice constants of Pnma-GaN are $a=$ $5.900 \AA, b=3.232 \AA$, and $c=5.374 \AA$ with LDA. The crystal structure of Pnma-GaN is shown in Figure 1(a). There are eight atoms in conventional cell, including four Ga atoms and four $\mathrm{N}$ atoms. There is an equivalent position for $\mathrm{Ga}$ or $\mathrm{N}$ atoms; that is to say, the four $\mathrm{Ga}$ atoms or $\mathrm{N}$ atoms are equivalent. Hence, it is reasonable that $\mathrm{Al}$ atom can replace any $\mathrm{Ga}$ atom. The Ga atoms occupy the crystallographic $4 c$ sites in a conventional cell, which is $(-0.1673,0.2500$, and 0.4008 ); and the $\mathrm{N}$ atoms occupy the crystallographic $4 c$ sites in a conventional cell, which is $(0.1731,0.2500$, and $0.4016)$, respectively. The crystal structure of $\mathrm{Ga}_{1-x} \mathrm{Al}_{x} \mathrm{~N}(x=$ $0.031,0.063,0.094$, and 0.125 ) is shown in Figures $1(\mathrm{~b}), 1(\mathrm{c})$, $1(\mathrm{~d})$, and $1(\mathrm{e})$, respectively. In $\mathrm{Ga}_{1-x} \mathrm{Al}_{x} \mathrm{~N}$ alloys, aluminum atoms substituted position is the lowest energy substitution method. In addition, wurtzite-GaN results were calculated using the same method; together with previous experimental results obtained other calculated results are presented in Table 1. The calculated lattice constants wurtzite-GaN (space group: $\left.\mathrm{P}_{3} \mathrm{mc}\right)$ are $a=b=3.156 \AA(3.225 \AA), c=5.148 \AA$ $(5.257 \AA)$, and internal parameter $u=0.376(0.375)$ with LDA (GGA), respectively. In addition, the experimental results of wurtzite-GaN are $a=b=3.190 \AA, c=5.189 \AA$ [23], and $u$ $=0.376$. The calculated lattice parameters of wurtzite-GaN are in good agreement with the reported calculated results of other theoretical results [23-26], while larger deviations 


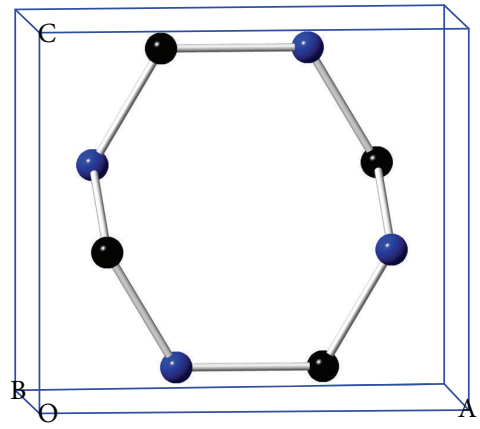

(a) $\mathrm{GaN}$

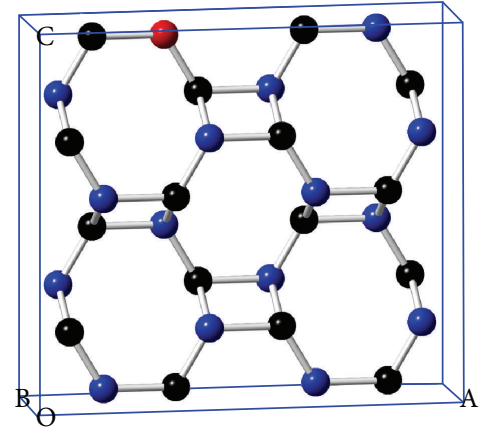

(b) $\mathrm{Ga}_{0.969} \mathrm{Al}_{0.031} \mathrm{~N}(2 \times 1 \times 2)$

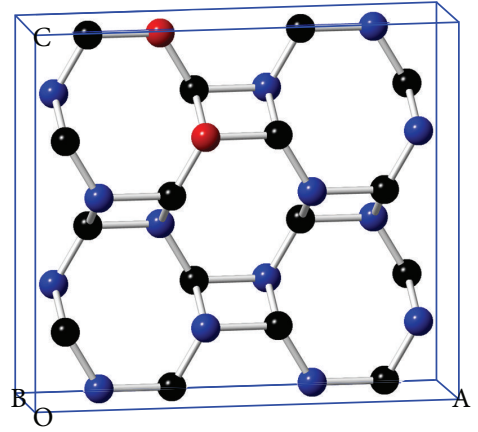

(c) $\mathrm{Ga}_{0.906} \mathrm{Al}_{0.063} \mathrm{~N}(2 \times 1 \times 2)$

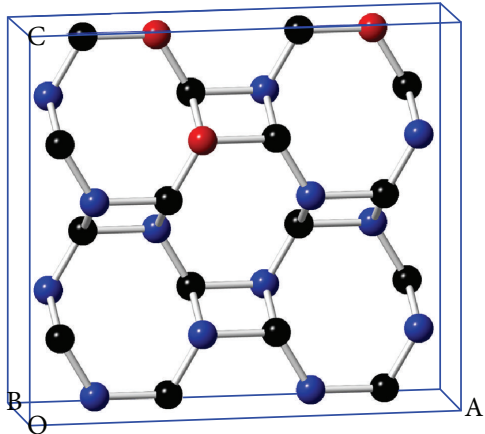

(d) $\mathrm{Ga}_{0.937} \mathrm{Al}_{0.094} \mathrm{~N}(2 \times 1 \times 2)$

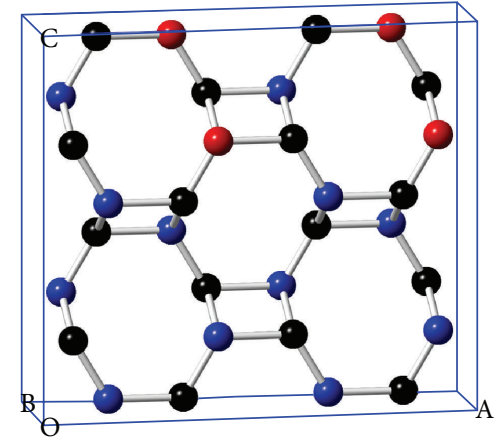

(e) $\mathrm{Ga}_{0.875} \mathrm{Al}_{0.125} \mathrm{~N}(2 \times 1 \times 2)$

Figure 1: Crystal structures of (a) Pnma-GaN, (b) $\mathrm{Ga}_{0.969} \mathrm{Al}_{0.031} \mathrm{~N}$, (c) $\mathrm{Ga}_{0.937} \mathrm{Al}_{0.063} \mathrm{~N}$, (d) $\mathrm{Ga}_{0.906} \mathrm{Al}_{0.094} \mathrm{~N}$, and (e) $\mathrm{Ga}_{0.875} \mathrm{Al}_{0.125} \mathrm{~N}$; the red, blue, and black spheres represent $\mathrm{Al}, \mathrm{Ga}$, and $\mathrm{N}$ atoms, respectively.

from experimental results are found in both $a$ and $c$ directions $1.10 \%$ and $3.11 \%$ with GGA, respectively, but smaller deviations are found in both $a$ and $c$ directions $1.07 \%$ and $0.79 \%$ with LDA, respectively. Due to the structural porous feature (see Figures 1(b)-1(e)), Pnma-GaN can also be expected to be a good hydrogen storage material. At ambient pressure, the enthalpy of Pnma-GaN is slightly greater $0.264 \mathrm{eV}$ per formula unit than that of wurtzite-GaN.

The elastic properties of a solid are important. They are not only closely related to various fundamental solid-state phenomena such as interatomic bonding, equations of state, and phonon spectra, but also linked thermodynamically with specific heat, thermal expansion, Debye temperature, and Grüneisen parameter. Most importantly, knowledge of the elastic constants is essential for many practical applications related to the mechanical properties of a solid. The criteria for mechanical stability of orthorhombic phase are given by [27]:

$$
\begin{aligned}
C_{i i} & >0 \\
{\left[C_{11}+C_{22}+C_{33}+2\left(C_{12}+C_{13}+C_{23}\right)\right]>0 } & \\
\left(C_{11}+C_{22}-2 C_{12}\right) & >0 \\
\left(C_{11}+C_{33}-2 C_{13}\right) & >0 \\
\left(C_{22}+C_{33}-2 C_{23}\right) & >0 .
\end{aligned}
$$

Obviously, the elastic constants of Pnma-GaN satisfy the generalized elastic stability criteria for a stable orthorhombic symmetry. To ensure the stability of Pnma-GaN, the phonon spectra were calculated at ambient conditions (see Figure 2(a)). There is no imaginary frequency, which means Pnma-GaN can be stable at ambient conditions. Calculated single crystal elastic constants $C_{i j}$, bulk modulus $B$, shear modulus $G$, and Young's modulus $E$ for Pnma and wurtzite phases of GaN are listed in Table 2. The calculated elastic constants of wurtzite-GaN are in excellent agreement with the reported calculated results of other theoretical results $[28,35]$. The calculated related enthalpies of Pnma-GaN, wurtzite-GaN, zinc blende-GaN, and $\mathrm{NaCl}-\mathrm{GaN}$ are shown in Figure 2(b). All of them are smaller than the enthalpy of $\alpha-\mathrm{Ga}$ and $\alpha-\mathrm{N}_{2}$. That is to say, they can be synthesized in the experiment.

The Debye temperature $\left(\Theta_{D}\right)$ is a fundamental physical property and correlates with many physical properties of solids, such as specific heat and the thermal coefficient [36]. It is used to distinguish between high and low temperature regions for a solid. If the temperature $T>\Theta_{D}$, we expect all modes to have the energy of $k_{B} T$, and if $T<\Theta_{D}$, one expects the high-frequency modes to be frozen; that is, the vibrational excitations arise solely from the acoustic vibrations. The standard formula to evaluate the Debye temperature is given by [37]:

$$
\Theta_{D}=\frac{h}{k_{B}}\left[\frac{3 n}{4 \pi}\left(\frac{N_{A} \rho}{M}\right)\right]^{1 / 3} v_{m},
$$




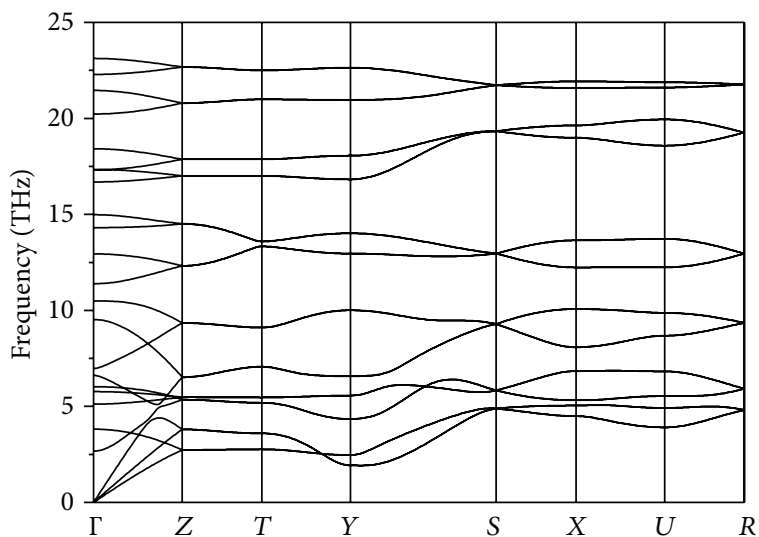

(a)

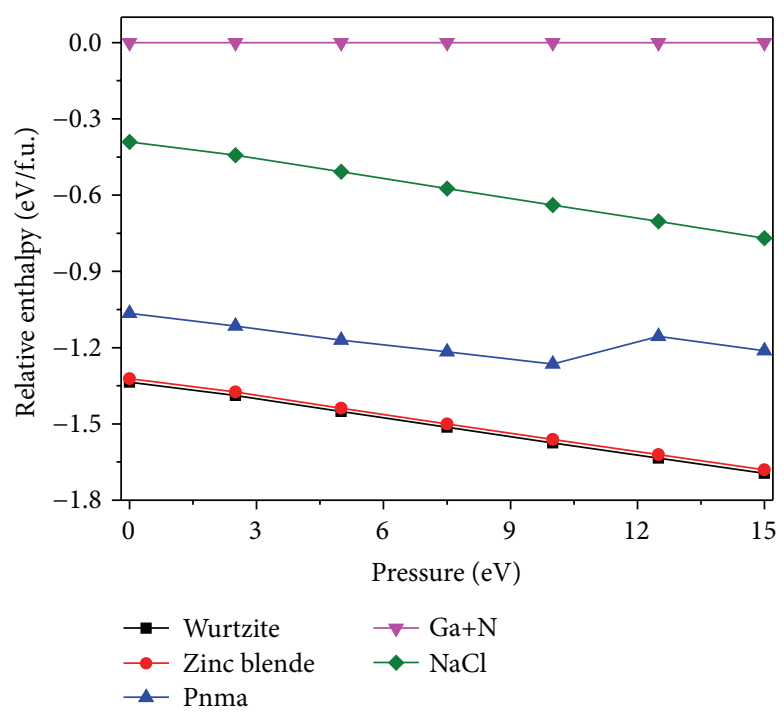

(b)

Figure 2: The phonon spectra of Pnma-GaN at ambient pressure (a); the related enthalpies of Pnma-GaN, wurtzite-GaN, zinc blende-GaN, and $\mathrm{NaCl}-\mathrm{GaN}(\mathrm{b})$.

TABLe 2: Calculated elastic constant $C_{i j}(\mathrm{GPa})$, bulk modulus $B(\mathrm{GPa})$, shear modulus $G(\mathrm{GPa})$, and Young's modulus $E$ (GPa) of GaN.

\begin{tabular}{cccccccccccccc}
\hline & & $C_{11}$ & $C_{12}$ & $C_{13}$ & $C_{22}$ & $C_{23}$ & $C_{33}$ & $C_{44}$ & $C_{55}$ & $C_{66}$ & $B$ & $G$ & $E$ \\
\hline \multirow{2}{*}{ Pnma } & $\mathrm{GGA}^{\mathrm{a}}$ & 156 & 63 & 114 & 271 & 117 & 263 & 72 & 92 & 66 & 134 & 66 & 170 \\
& $\mathrm{LDA}^{\mathrm{a}}$ & 147 & 80 & 141 & 315 & 145 & 306 & 75 & 97 & 64 & 150 & 68 & 177 \\
\hline \multirow{6}{*}{$\mathrm{P}_{3} \mathrm{mc}$} & $\mathrm{GGA}^{\mathrm{a}}$ & 321 & 107 & 84 & & & 334 & 88 & & 107 & 170 & 103 & 257 \\
& $\mathrm{LDA}^{\mathrm{a}}$ & 372 & 137 & 107 & & & 425 & 96 & & 118 & 208 & 115 & 291 \\
& $\mathrm{LDA}^{\mathrm{b}}$ & 334 & 132 & 99 & & & 372 & 86 & & 101 & 189 & 109 \\
& $\mathrm{LDA}^{\mathrm{c}}$ & 377 & 160 & 114 & & & 209 & 81 & & 109 & 330 \\
& $\mathrm{LDA}^{\mathrm{d}}$ & 396 & 144 & 100 & & & 392 & 91 & & 126 & & \\
& Exp. & $373^{\mathrm{f}}$ & 141 & 80 & & & 387 & 94 & & 118 & $207^{\mathrm{e}}$ \\
\hline
\end{tabular}

${ }^{\mathrm{a}}$ This work, ${ }^{\mathrm{b}}[23],{ }^{\mathrm{c}}[27],{ }^{\mathrm{d}}[28],{ }^{\mathrm{e}}[25]$, and ${ }^{\mathrm{f}}[29]$.

where $h$ is Planck's constant, $k_{B}$ is Boltzmann's constant, $n$ is the number of atoms per formula unit, and $N_{A}, M$, and $\rho$ represent Avogadro's number, molar mass, and density, respectively. The average sound velocity $v_{m}$ is described by the following expression:

$$
v_{m}=\left[\frac{1}{3}\left(\frac{2}{v_{t}^{3}}+\frac{1}{v_{l}^{3}}\right)\right]^{-1 / 3} .
$$

The transverse and longitudinal elastic wave velocity, $v_{t}$ and $v_{l}$, can be achieved from Navier's equation as follows:

$$
\begin{aligned}
& v_{t}=\sqrt{\frac{G}{\rho},} \\
& v_{l}=\sqrt{\frac{3 B+4 G}{3 \rho}},
\end{aligned}
$$

where $B$ and $G$ are bulk modulus and shear modulus, respectively. The calculated Debye temperature $\Theta_{D}=511 \mathrm{~K}$ is evaluated from (2). The Debye temperature of Pnma-GaN is slightly smaller than that of wurtzite-GaN $(600 \mathrm{~K})$.
It is well known that the anisotropy of elasticity is an important implication in engineering science and crystal physics. The directional dependence of Young's modulus and the projection in $x y, x z$, and $y z$ planes for Pnma-GaN are illustrated in Figures 3(a) and 3(b). The maximum value of $E_{\text {max }}=221 \mathrm{GPa}$ and the minimal of $E_{\min }=106 \mathrm{GPa}$. The average value of all directions is $176 \mathrm{GPa}$, which is very close to CASTEP value (170 GPa; see Table 2$)$. The ratio $E_{\max } / E_{\min }$ = 2.09 shows Pnma-GaN exhibits a large elastic anisotropy. The directional dependence of Young's modulus and the projection in $x y, x z$, and $y z$ planes for wurtzite-GaN are shown in Figures 3(c) and 3(d). The maximal value of $E_{\max }$ $=301 \mathrm{GPa}$ and the minimal value of $E_{\min }=235 \mathrm{GPa}$. Its CASTEP value is $257 \mathrm{GPa}$, and this value is smaller than the average value of all directions by $1.15 \%$ (260 GPa; see Table 2). The ratio of $E_{\max } / E_{\min }$ is 1.28 ; it shows that wurtzite-GaN exhibits a smaller elastic anisotropy than that of Pnma-GaN.

The electronic band structure of Pnma-GaN is shown in Figure 4(a). The dashed line represents the Fermi level $\left(E_{F}\right)$. The coordinate of high symmetry points in Brillouin zone for Pnma-GaN is $\Gamma\left(\begin{array}{lll}0.0 & 0.0 & 0.0\end{array}\right), Z\left(\begin{array}{lll}0.0 & 0.0 & 0.5\end{array}\right)$, 

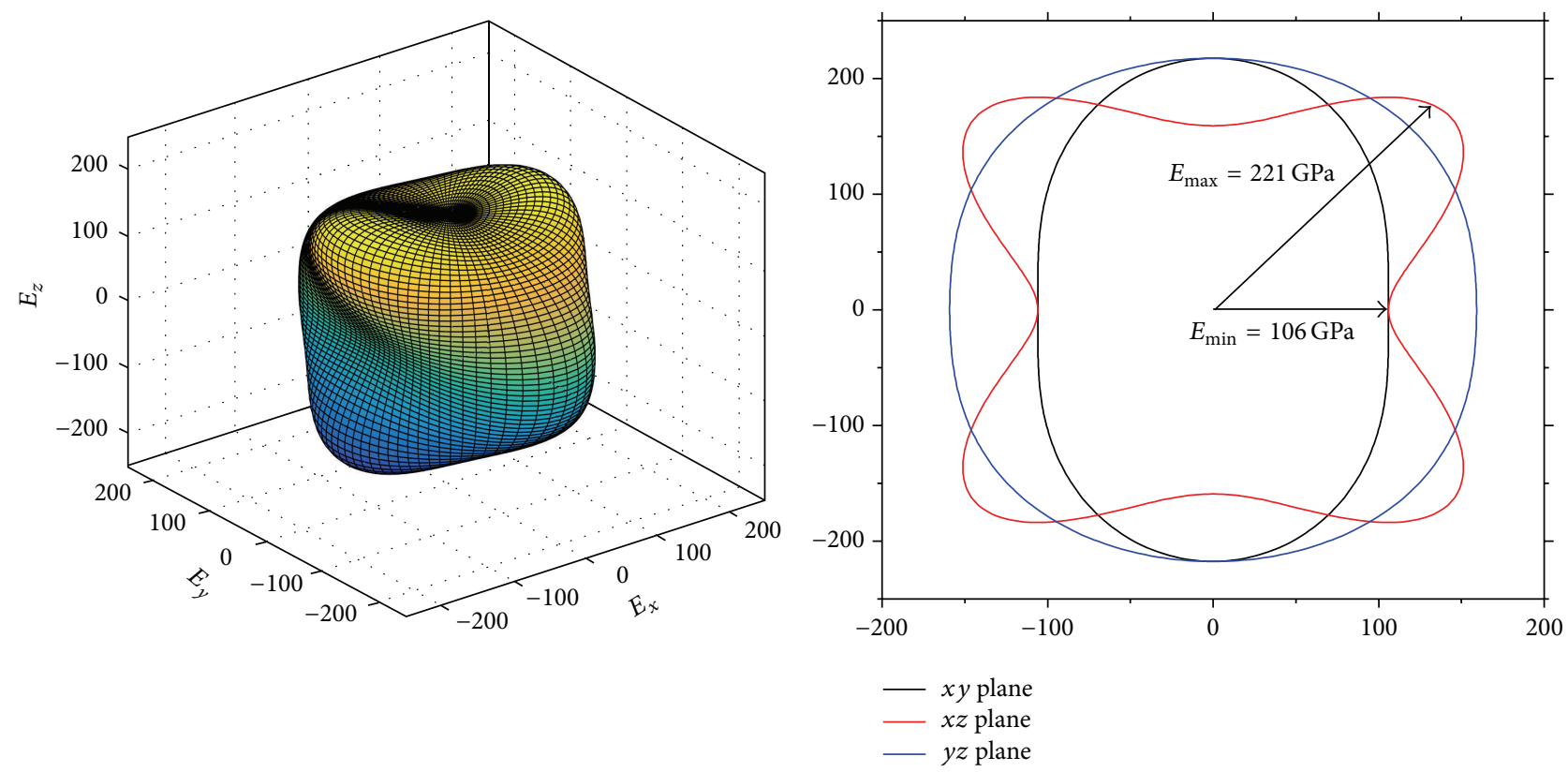

(a) Pnma

(b) Pnma
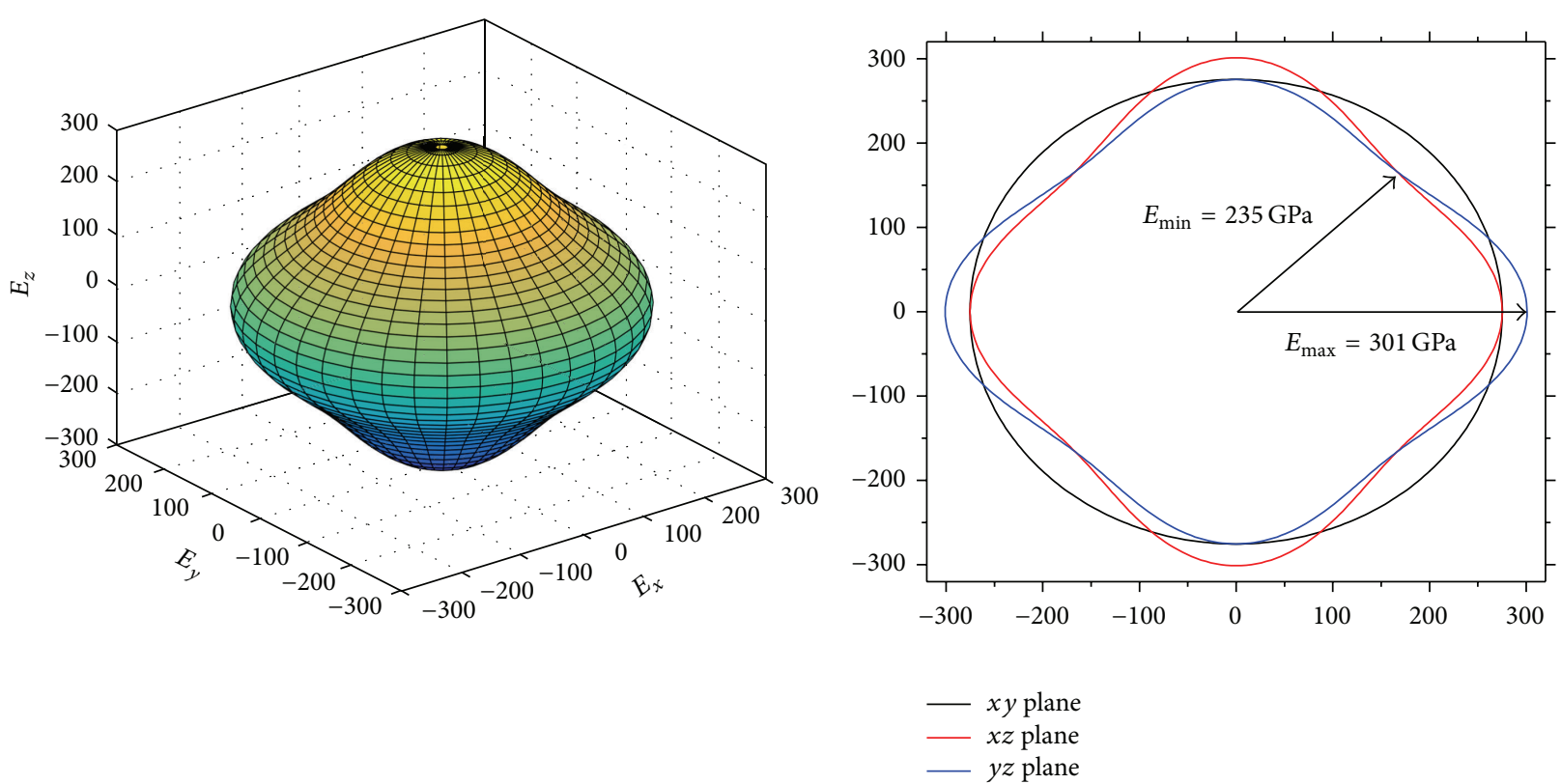

(c) $\mathrm{P}_{3} \mathrm{mc}$

(d) $\mathrm{P}_{3} \mathrm{mc}$

Figure 3: The directional dependence of Young's modulus for Pnma-GaN (a), 2D representation of Young's modulus for Pnma-GaN in the $x y$ plane, $x z$ plane, and $y z$ plane (b). The directional dependence of Young's modulus for wurtzite-GaN (c), $2 \mathrm{D}$ representation of Young's modulus for wurtzite-GaN in the $x y$ plane, $x z$ plane, and $y z$ plane (d). The units of axes are all in GPa.

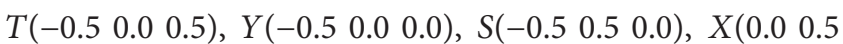
$0.0), U(0.00 .50 .5)$, and $R(-0.50 .50 .5)$. From the electronic band structure, it is shown that Pnma-GaN is a direct semiconductor and its band gap is $1.567 \mathrm{eV}$. It is known that the calculated band gap with DFT is usually underestimated by $30-50 \%$, or even more; the true band gap must be larger than the calculated results. The valence band maximum and conduction band minimum are both at $\Gamma$ point. The total density of states and the partial wave density of states projected onto different atoms at ambient pressure are also shown in Figure 4. The main features of Pnma-GaN can be illustrated as follows: (i) the part present in the energy part (2.5 to $12.5 \mathrm{eV}$ ) of the DOS is mainly due to contributions from the $p$ electrons and $s$ electrons of $\mathrm{Ga}$ atom, and the contribution of the $\mathrm{N}$ atom $s$ orbital is the smallest; (ii) the states from $-7.5 \mathrm{eV}$ to Fermi energy mainly originate from $p$ electrons of $\mathrm{N}$ atom and with slight contributions from $s$ electrons of $\mathrm{N}$ atom; (iii) the peak present in the lower 


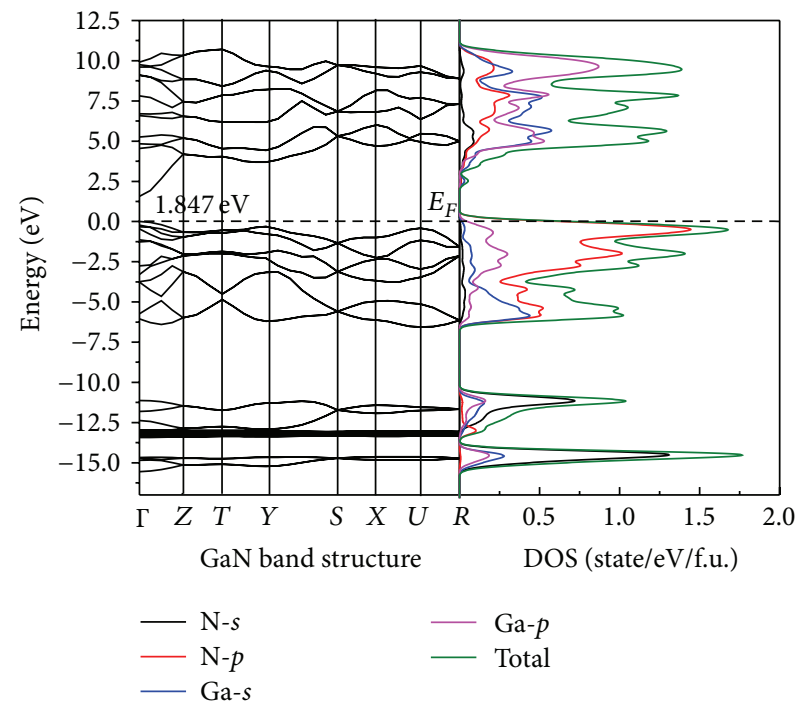

(a)

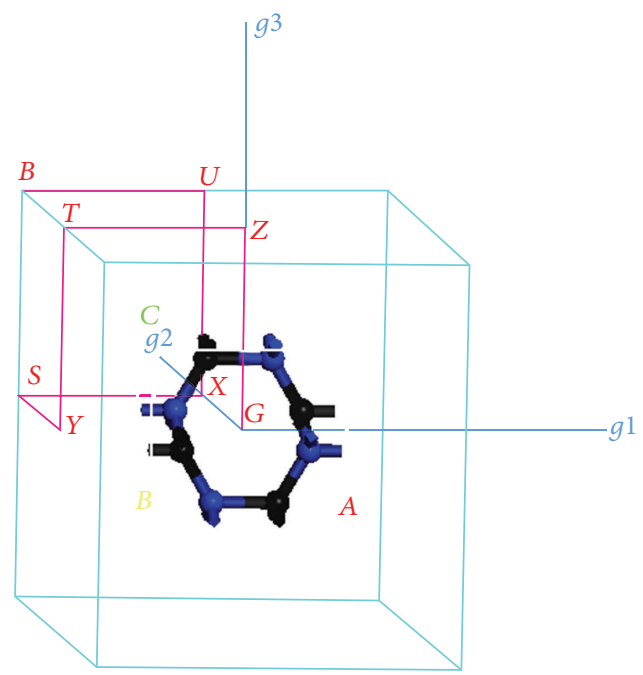

(b)

FIGURE 4: Electronic band structure and density of state (a) for the Pnma-GaN at ambient pressure and Brillouin zone of Pnma-GaN (b).

energy part $(-17.5$ to $-10 \mathrm{eV})$ of the DOS is mainly due to contributions from the $s$ electrons of $\mathrm{N}$, and the contribution from $\mathrm{N}-p$ is very small.

The calculated band structures of $\mathrm{Ga}_{1-x} \mathrm{Al}_{x} \mathrm{~N}(x=0.031$, $0.063,0.094$, and 0.125) for Pnma and wurtzite phases are plotted in Figures 5(a)-5(d) and 5(e)-5(h), respectively. The coordinate of high symmetry points in Brillouin zone for Pnma- $\mathrm{Ga}_{1-x} \mathrm{Al}_{x} \mathrm{~N}$ is $\Gamma\left(\begin{array}{lll}0.0 & 0.0 & 0.0\end{array}\right), F\left(\begin{array}{lll}0.0 & 0.5 & 0.0\end{array}\right)$, $Q\left(\begin{array}{lll}0.0 & 0.5 & 0.5\end{array}\right)$, and $Z\left(\begin{array}{lll}0.0 & 0.0 & 0.5\end{array}\right)$. The dashed line also represents the Fermi level $\left(E_{F}\right)$. Calculating the band structures of $\mathrm{Ga}_{1-x} \mathrm{Al}_{x} \mathrm{~N}$, aluminum atoms substituted position is the lowest energy substitution method. The values of band gap as a function of concentration for Pnma- $\mathrm{Ga}_{1-x} \mathrm{Al}_{x} \mathrm{~N}$ and wurtzite- $\mathrm{Ga}_{1-x} \mathrm{Al}_{x} \mathrm{~N}$ are listed in Table 3. Similarly, it is recognized within DFT that the energy band gap of electronic structure calculations is commonly underestimated for both GGA and LDA, while the LDA values are close to the experimental values for wurtzite-GaN, so we show the band structures of Pnma- $\mathrm{Ga}_{1-x} \mathrm{Al}_{x} \mathrm{~N}$ and wurtzite- $\mathrm{Ga}_{1-x} \mathrm{Al}_{x} \mathrm{~N}$ in Figure 5 with LDA. This is generally due to the circumstance that they have sample forms that are not adequately flexible to precisely reproduce both the exchange-correlation energy and equivalent potential [31]. The electronic energy band structure of $\mathrm{Ga}_{0.969} \mathrm{Al}_{0.031} \mathrm{~N}, \mathrm{Ga}_{0.937} \mathrm{Al}_{0.063} \mathrm{~N}, \mathrm{Ga}_{0.906} \mathrm{Al}_{0.094} \mathrm{~N}$, and $\mathrm{Ga}_{0.875} \mathrm{Al}_{0.125} \mathrm{~N}$ for Pnma phase shows that they are direct semiconductors and its band gap is $1.740,1.640$, 1.710 , and $1.741 \mathrm{eV}$, respectively. Furthermore, the electronic energy band structure of $\mathrm{Ga}_{0.969} \mathrm{Al}_{0.031} \mathrm{~N}, \mathrm{Ga}_{0.937} \mathrm{Al}_{0.063} \mathrm{~N}$, $\mathrm{Ga}_{0.906} \mathrm{Al}_{0.094} \mathrm{~N}$, and $\mathrm{Ga}_{0.875} \mathrm{Al}_{0.125} \mathrm{~N}$ for wurtzite phase shows that they are direct semiconductors and its band gap is $2.217,2.327,2.489$, and $2.648 \mathrm{eV}$, respectively. With the same composition $x$, the band gap of $\mathrm{Ga}_{1-x} \mathrm{Al}_{x} \mathrm{~N}$ alloy for Pnma phase is smaller than that of wurtzite phase. With the
TABLE 3: Calculated band gap (eV) for $\mathrm{Ga}_{1-x} \mathrm{Al}_{x} \mathrm{~N}$ alloy.

\begin{tabular}{lccccc}
\hline Space group & \multicolumn{2}{c}{ Pnma } & \multicolumn{2}{c}{$\mathrm{P}_{3} \mathrm{mc}$} & \\
\hline$x$ & $\mathrm{GGA}^{\mathrm{a}}$ & $\mathrm{LDA}^{\mathrm{a}}$ & $\mathrm{GGA}^{\mathrm{a}}$ & $\mathrm{LDA}^{\mathrm{a}}$ & Other works \\
0 & 1.567 & 1.847 & 1.889 & 2.080 & $3.390^{\mathrm{b}, \mathrm{c}, \mathrm{d}, \mathrm{f}}, 3.470^{\mathrm{e}}$ \\
0.031 & & 1.740 & & 2.217 & \\
0.063 & & 1.640 & & 2.327 & \\
0.094 & & 1.710 & & 2.489 & \\
0.125 & & 1.741 & & 2.648 & \\
\hline
\end{tabular}

${ }^{\mathrm{a}}$ This work, ${ }^{\mathrm{b}}[30],{ }^{\mathrm{c}}[31],{ }^{\mathrm{d}}[32],{ }^{\mathrm{e}}[33]$, and ${ }^{\mathrm{f}}[34]$.

increasing composition $x$, the band gap decreases initially and increases afterward for Pnma- $\mathrm{Ga}_{1-x} \mathrm{Al}_{x} \mathrm{~N}$, while, for wurtzite$\mathrm{Ga}_{1-x} \mathrm{Al}_{x} \mathrm{~N}$, the band gap increases with the composition $x$ increasing.

\section{Conclusions}

This study reports a detailed investigation of the structural and electronic properties of Pnma-GaN/ $\mathrm{Ga}_{1-x} \mathrm{Al}_{x} \mathrm{~N}$ semiconductor alloy using the DFT within the ultrasoft pseudopotential scheme in the frame of LDA and GGA. The elastic constants and phonon calculations reveal Pnma-GaN is mechanically and dynamically stable at ambient pressure and its Debye temperature is $510.55 \mathrm{~K}$. Pnma-GaN is a direct semiconductor and its band gap is $1.847 \mathrm{eV}$ within LDA at ambient pressure. The elastic anisotropy properties show that Pnma-GaN exhibits a large anisotropy in its Young's modulus. When the composition of aluminum increases from 0 to 0.125 in the alloys, the band gap decreases initially and increases afterwards for Pnma- $\mathrm{Ga}_{1-x} \mathrm{Al}_{x} \mathrm{~N}$, while, for 


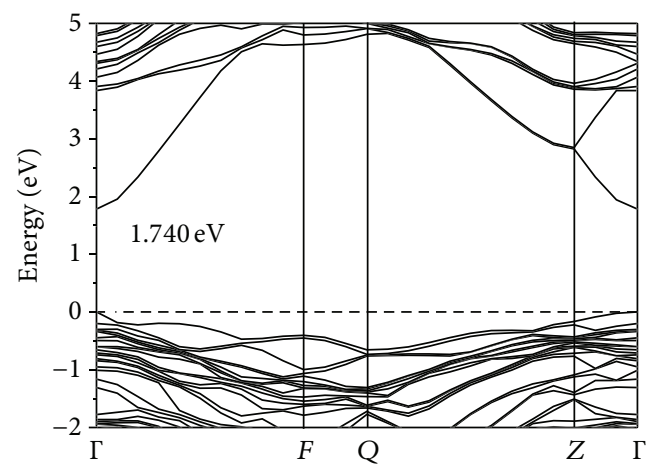

(a) $\mathrm{Ga}_{0.969} \mathrm{Al}_{0.031} \mathrm{~N}$ band structure (Pnma)

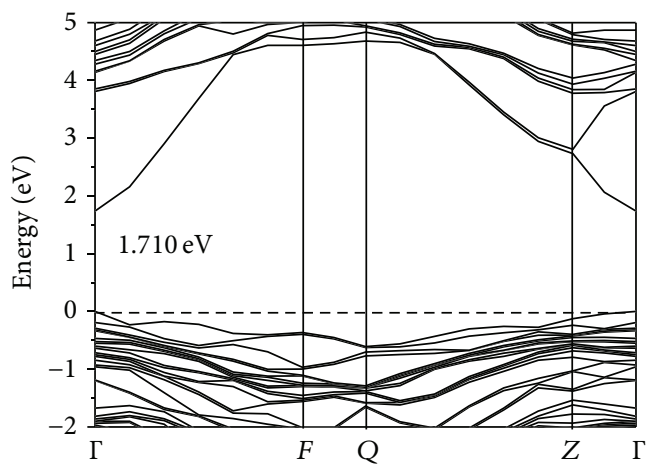

(c) $\mathrm{Ga}_{0.906} \mathrm{Al}_{0.094} \mathrm{~N}$ band structure (Pnma)

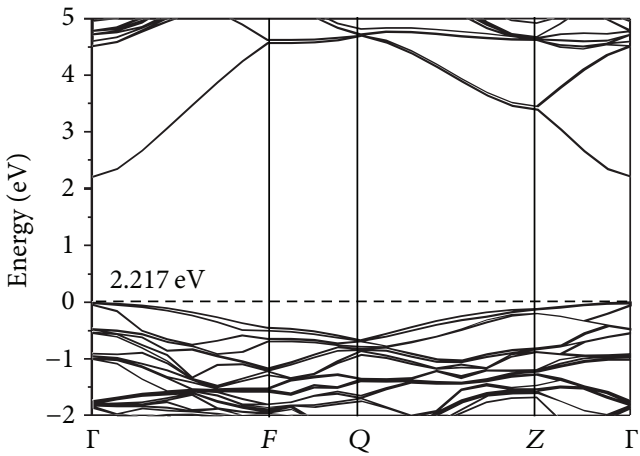

(e) $\mathrm{Ga}_{0.969} \mathrm{Al}_{0.031} \mathrm{~N}$ band structure $\left(\mathrm{P6}_{3} \mathrm{mc}\right)$

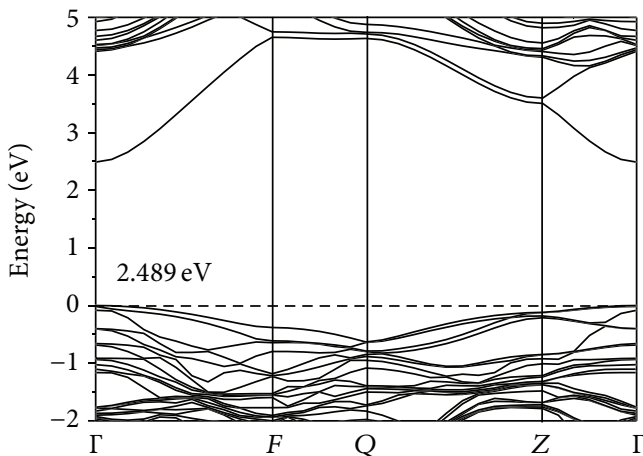

(g) $\mathrm{Ga}_{0.906} \mathrm{Al}_{0.094} \mathrm{~N}$ band structure $\left(\mathrm{P}_{3} \mathrm{mc}\right)$

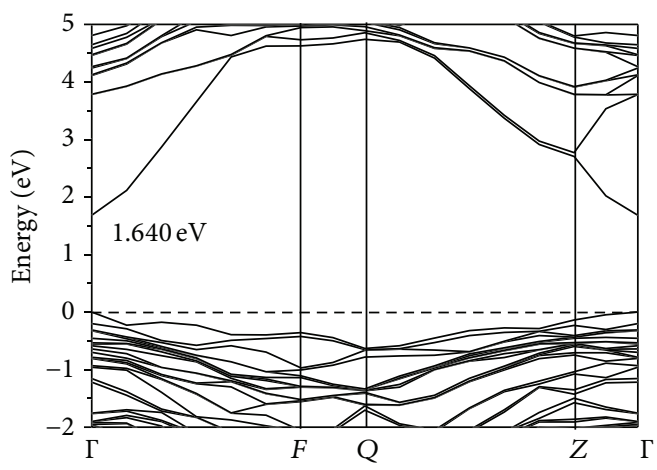

(b) $\mathrm{Ga}_{0.937} \mathrm{Al}_{0.063} \mathrm{~N}$ band structure (Pnma)

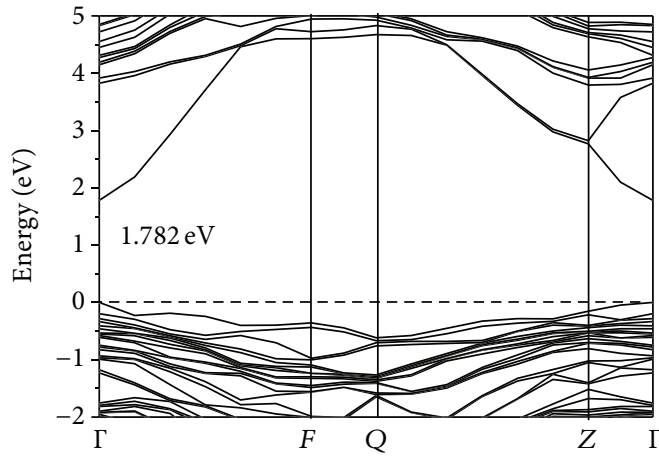

(d) $\mathrm{Ga}_{0.875} \mathrm{Al}_{0.125} \mathrm{~N}$ band structure (Pnma)

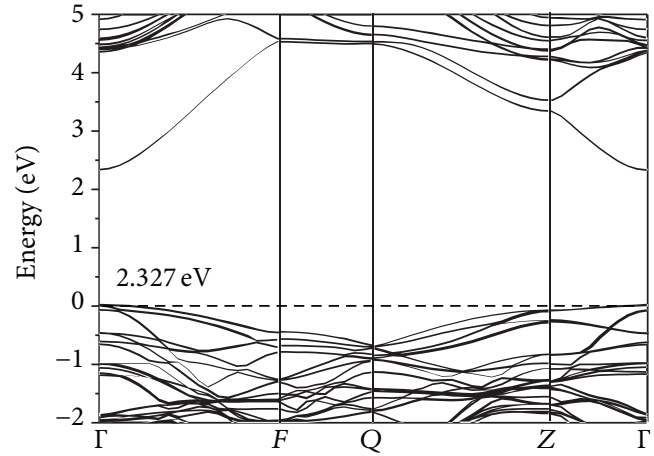

(f) $\mathrm{Ga}_{0.937} \mathrm{Al}_{0.063} \mathrm{~N}$ band structure $\left(\mathrm{P}_{3} \mathrm{mc}\right)$

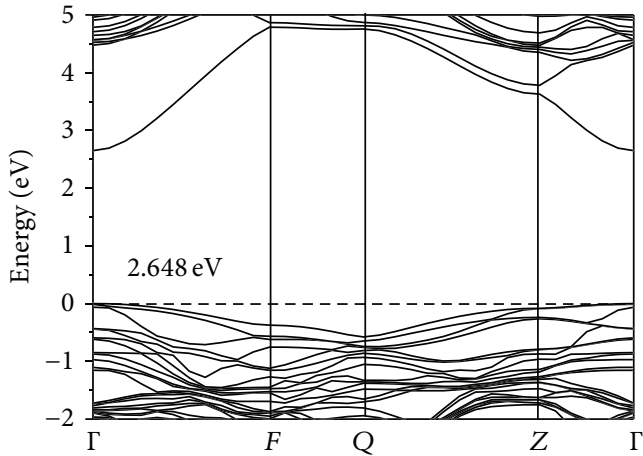

(h) $\mathrm{Ga}_{0.875} \mathrm{Al}_{0.125} \mathrm{~N}$ band structure $\left(\mathrm{P}_{3} \mathrm{mc}\right)$

FIGURE 5: Electronic band structures of the $\mathrm{Ga}_{0.969} \mathrm{Al}_{0.031} \mathrm{~N}(\mathrm{a}), \mathrm{Ga}_{0.937} \mathrm{Al}_{0.063} \mathrm{~N}$ (b), $\mathrm{Ga}_{0.906} \mathrm{Al}_{0.094} \mathrm{~N}$ (c), and $\mathrm{Ga}_{0.875} \mathrm{Al}_{0.125} \mathrm{~N}(\mathrm{~d})$ for Pnma-GaN at ambient pressure. Electronic band structures of the $\mathrm{Ga}_{0.969} \mathrm{Al}_{0.031} \mathrm{~N}(\mathrm{e}), \mathrm{Ga}_{0.937} \mathrm{Al}_{0.063} \mathrm{~N}(\mathrm{f}), \mathrm{Ga}_{0.906} \mathrm{Al}_{0.094} \mathrm{~N}(\mathrm{~g})$, and $\mathrm{Ga}_{0.875} \mathrm{Al}_{0.125} \mathrm{~N}(\mathrm{~h})$ for wurtzite-GaN at ambient pressure. 
wurtzite- $\mathrm{Ga}_{1-x} \mathrm{Al}_{x} \mathrm{~N}$, the band gap increases as the composition $x$ increases. Due to the structural porous feature, Pnma$\mathrm{GaN}$ can also be expected to be a good hydrogen storage material.

\section{Competing Interests}

The authors declare that they have no competing interests.

\section{Acknowledgments}

This work was supported by the Natural Science Foundation of China (no. 61474089) and Open Fund of Key Laboratory of Complex Electromagnetic Environment Science and Technology, China Academy of Engineering Physics (no. 20150214. XY.K).

\section{References}

[1] W. Kohn and L. J. Sham, "Self-consistent equations including exchange and correlation effects," Physical Review A, vol. 140, pp. A1133-A1138, 1965.

[2] E. Przeździecka, K. Gościński, M. Stachowicz et al., "Spectrum selective UV detectors from an $p-\mathrm{ZnO}: \mathrm{As} / \mathrm{n}-\mathrm{GaN}$ diodes grown by Molecular Beam Epitaxy," Sensors and Actuators A: Physical, vol. 195, pp. 27-31, 2014.

[3] X. D. Wang, W. D. Hu, M. Pan et al., "Study of gain and photoresponse characteristics for back-illuminated separate absorption and multiplication GaN avalanche photodiodes," Journal of Applied Physics, vol. 115, Article ID 013103, 2014.

[4] A. Rubio, J. L. Corkill, and M. L. Cohen, "Quasiparticle band structures of short-period superlattices and ordered alloys of AlN and GaN," Physical Review B, vol. 49, no. 3, pp. 1952-1956, 1994.

[5] M. Malachowski, I. R. Kityk, and B. Sahraoui, "Electronic structure and optical response in $\mathrm{Ga}_{x} \mathrm{Al}_{1-x} \mathrm{~N}$ solid alloys," Physics Letters A, vol. 242, no. 6, pp. 337-342, 1998.

[6] I. Kityk, "Band structure of wurtzite-like $\mathrm{Ga}_{x} \mathrm{Al}_{1-x} \mathrm{~N}$ solid state alloys," Materials Letters, vol. 57, no. 12, pp. 1798-1804, 2003.

[7] M. Malachowski, I. R. Kityk, and B. Sahraoui, "Band structure and optical response in $\mathrm{Ga}_{x} \mathrm{Al}_{1-x} \mathrm{~N}$," Physica Status Solidi B, vol. 207, no. 2, pp. 405-418, 1998.

[8] H. Okumura, S. Yoshida, and T. Okahisa, "Optical properties near the band gap on hexagonal and cubic GaN," Applied Physics Letters, vol. 64, no. 22, pp. 2997-2999, 1994.

[9] K. Miwa and A. Fukumoto, "First-principles calculation of the structural, electronic, and vibrational properties of gallium nitride and aluminum nitride," Physical Review B, vol. 48, no. 11, pp. 7897-7902, 1993.

[10] J. L. P. Hughes, Y. Wang, and J. E. Sipe, "Calculation of linear and second-order optical response in wurtzite GaN and AlN," Physical Review B, vol. 55, no. 20, pp. 13630-13640, 1997.

[11] Y. Xu and W. Y. Ching, "Electronic, optical, and structural properties of some wurtzite crystals," Physical Review B, vol. 48, article 4335, 1993.

[12] P. Hohenberg and W. Kohn, "Inhomogeneous electron gas," Physical Review Letters, vol. 136, no. 3, p. B864, 1964.

[13] J. P. Perdew, K. Burke, and M. Ernzerhof, "Generalized gradient approximation made simple," Physical Review Letters, vol. 77, no. 18 , pp. 3865-3868, 1996.
[14] J. P. Perdew and A. Zunger, "Self-interaction correction to density-functional approximations for many-electron systems," Physical Review B, vol. 23, no. 10, pp. 5048-5079, 1981.

[15] D. M. Ceperley and B. J. Alder, "Ground state of the electron gas by a stochastic method," Physical Review Letters, vol. 45, no. 7, pp. 566-569, 1980.

[16] S. J. Clark, M. D. Segall, C. J. Pickard et al., "First principles methods using CASTEP," Zeitschrift für Kristallographie, vol. 220, no. 5-6, pp. 567-570, 2005.

[17] H. J. Monkhorst and J. D. Pack, "Special points for Brillouinzone integrations," Physical Review B: Solid State, vol. 13, no. 12, pp. 5188-5192, 1976.

[18] B. G. Pfrommer, M. Côté, S. G. Louie, and M. L. Cohen, "Relaxation of crystals with the quasi-newton method," Journal of Computational Physics, vol. 131, no. 1, pp. 233-240, 1997.

[19] W. Voigt, Lehrburch der Kristallphysik, Teubner, Leipzig, Germany, 1928.

[20] A. Reuss, "Berechnung der fließgrenze von mischkristallen auf grund der plastizitätsbedingung für einkristalle," Zeitschrift für Angewandte Mathematik und Mechanik, vol. 9, no. 1, pp. 49-58, 1929.

[21] R. Hill, "The elastic behaviour of a crystalline aggregate," Proceedings of the Physical Society, Section A, vol. 65, no. 5, pp. 349-354, 1952.

[22] S. Baroni, S. de Gironcoli, A. dal Corso, and P. Giannozzi, "Phonons and related crystal properties from densityfunctional perturbation theory," Reviews of Modern Physics, vol. 73, no. 2, pp. 515-562, 2001.

[23] H. Schulz and K. H. Thiemann, "Crystal structure refinement of AlN and GaN," Solid State Communications, vol. 23, no. 11, pp. 815-819, 1977.

[24] Y. Duan, G. Tang, L. Qin, and L. Shi, "Elasticity and polarization of $\mathrm{Ga}_{\mathrm{x}} \mathrm{Al}_{1-\mathrm{x}} \mathrm{N}$ alloys subjected to uniaxial and biaxial compression," The European Physical Journal B, vol. 66, no. 2, pp. 211-215, 2008.

[25] F. Bernardini, V. Fiorentini, and D. Vanderbilt, "Spontaneous polarization and piezoelectric constants of III-V nitrides," Physical Review B-Condensed Matter and Materials Physics, vol. 56, no. 16, pp. R10024-R10027, 1997.

[26] J. M. Wagner and F. Bechstedt, "Properties of strained wurtzite GaN and AlN: Ab initio studies," Physical Review B, vol. 66, no. 11, Article ID 115202, 2002.

[27] J. F. Nye, Physical Properties of Crystals, Oxford University Press, 1985.

[28] O. Madelung, Semiconductors: Data Handbook, Springer, Berlin, Germany, 2004.

[29] T. Deguchi, D. Ichiryu, K. Toshikawa et al., "Structural and vibrational properties of GaN," Journal of Applied Physics, vol. 86, no. 4, pp. 1860-1866, 1999.

[30] M. Trivedi and K. Shenai, "Performance evaluation of highpower wide band-gap semiconductor rectifiers," Journal of Applied Physics, vol. 85, no. 9, pp. 6889-6897, 1999.

[31] S. Strite, "GaN, AlN, and InN: a review," Journal of Vacuum Science \& Technology B, vol. 10, article 1237, 1992.

[32] H. Morkoç, S. Strite, G. B. Gao, M. E. Lin, B. Sverdlov, and M. Burns, "Large-band-gap SiC, III-V nitride, and II-VI ZnSebased semiconductor device technologies," Journal of Applied Physics, vol. 76, no. 3, pp. 1363-1398, 1994.

[33] V. Bougrov, M. E. Levinshtein, S. L. Rumyantsev, and A. Zubrilov, Properties of Advanced Semiconductor Materials GaN, 
AlN, InN, BN, SiC, SiGe, John Wiley \& Sons, New York, NY, USA, 2001.

[34] T. P. Chow and M. Ghezzo, "SiC power devices," in III-Nitride, $\mathrm{SiC}$, and Diamond Materials for Electronic, D. K. Gaskill, C. D. Brandt, and R. J. Nemanich, Eds., vol. 423 of MRS Symposium Proceedings, pp. 69-73, Materials Research Society, Pittsburgh, $\mathrm{Pa}, \mathrm{USA}, 1996$.

[35] S. P. Wan, J. B. Xia, and K. Chang, "Effects of piezoelectricity and spontaneous polarization on electronic and optical properties of wurtzite III-V nitride quantum wells," Journal of Applied Physics, vol. 90, no. 12, article 6210, 2001.

[36] Y.-J. Hao, X.-R. Chen, H.-L. Cui, and Y.-L. Bai, "First-principles calculations of elastic constants of c-BN," Physica B: Condensed Matter, vol. 382, no. 1-2, pp. 118-122, 2006.

[37] O. L. Anderson, "A simplified method for calculating the debye temperature from elastic constants," Journal of Physics and Chemistry of Solids, vol. 24, no. 7, pp. 909-917, 1963. 

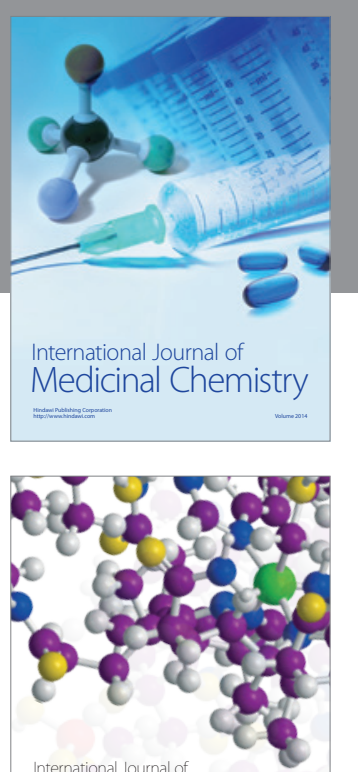

Carbohydrate Chemistry

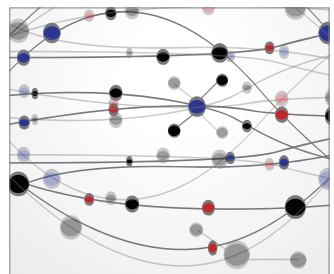

The Scientific World Journal
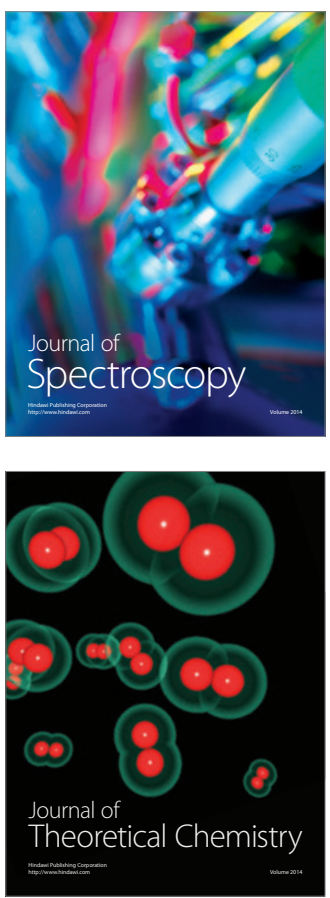
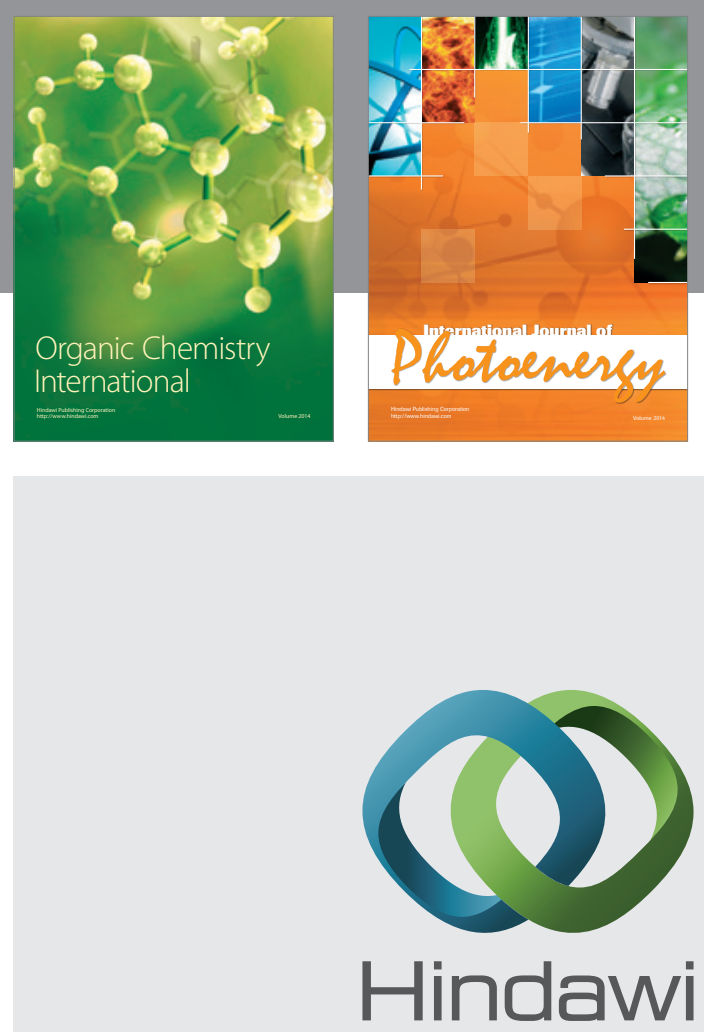

Submit your manuscripts at

http://www.hindawi.com

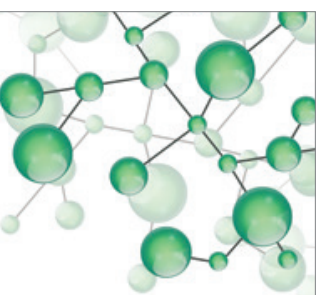

International Journal of

Inorganic Chemistry

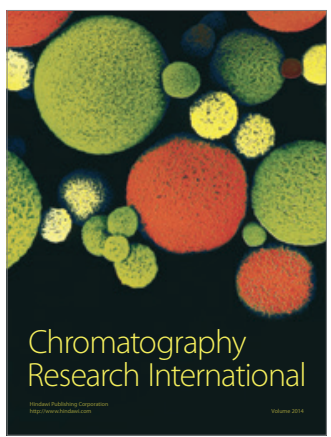

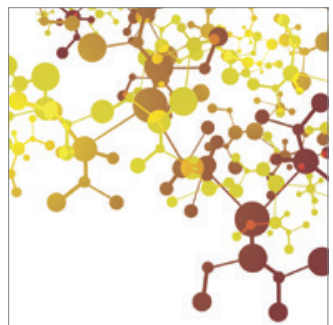

Applied Chemistry
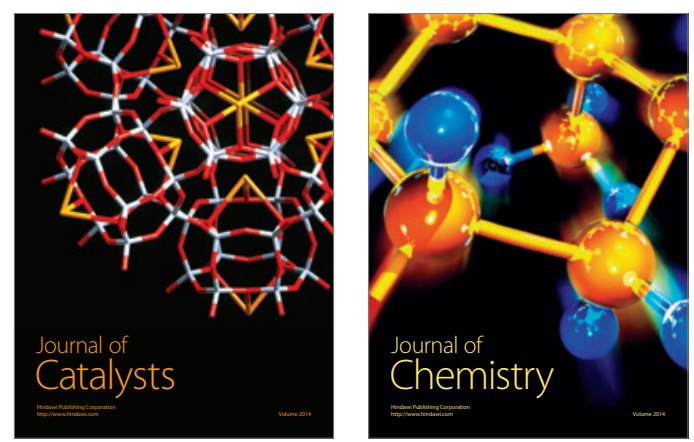
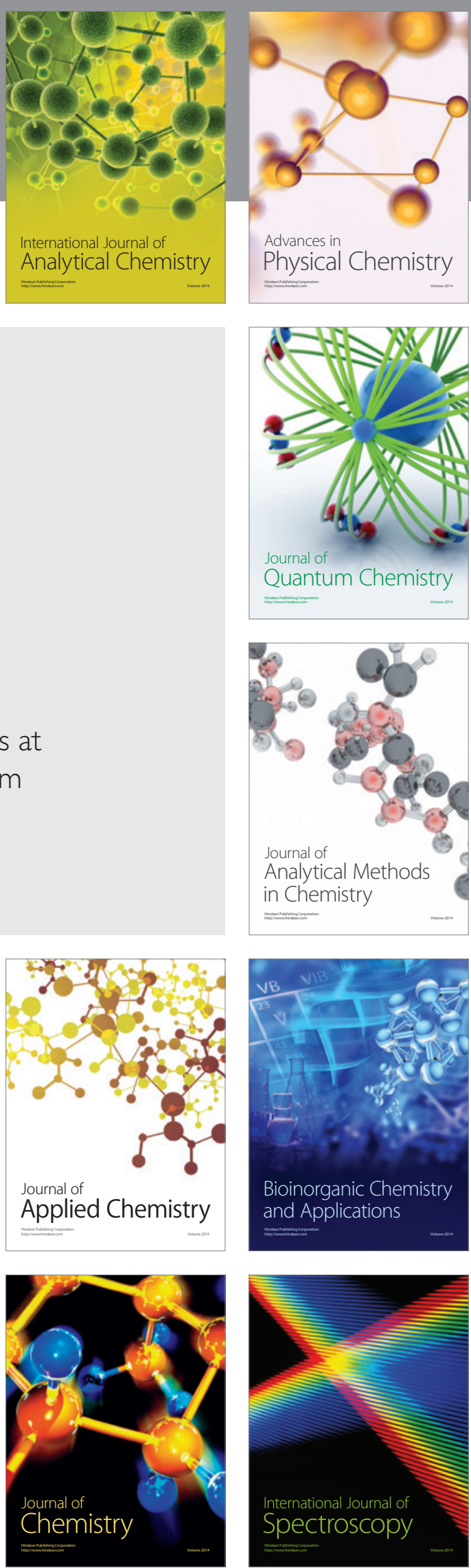